\title{
Preliminary Communication
}

\section{Gayane Tokmajyan and Lusine Karapetyan* \\ One-pot two-step synthesis of 2,5-dihydro-2- oxofuran-3-carboxamides}

\begin{abstract}
Dihydro-2-oxofuran-3-carboxamides were synthesized by a one-pot two-step reaction catalyzed by sodium methoxide. Readily available tertiary $\alpha$-hydroxyketones were condensed with substituted cyanoacetamides to give 2-imino-2,5-dihydrofuran-3-carboxamides that, without isolation, were hydrolized to the title products.
\end{abstract}

Keywords: acidic hydrolysis; $\alpha$-hydroxyketone; cyanoacetamide; 2,5-dihydro-2-oxofuran-3-carboxamide; 2-imino-2,5-dihydrofuran-3-carboxamide.

*Corresponding author: Lusine Karapetyan, Yerevan State University, Alex Manoogian 1, 0025, Yerevan, Armenia, e-mail: lousine_karapetyan@yahoo.com; lkarapetyan@ysu.am Gayane Tokmajyan: Yerevan State University, Alex Manoogian 1, 0025, Yerevan, Armenia

\section{Introduction}

2,5-Dihydro-2-oxofuran derivatives are a large family of heterocycles that include synthetically useful compounds, several natural products [1-14], and a number of drugs with diverse biological activities such as antifungal, antibacterial, and anti-inflammatory properties [15-19]. There has been a continuous interest in the development of efficient and convenient methods for the preparation of these heterocycles [10-14, 20-22]. In an extension of our synthetic studies on 2,5-dihydro-2-oxofurans, here we report a convenient and efficient synthesis of the title compounds starting from readily available tertiary $\alpha$-hydroxyketones 1a,b (Scheme 1).

The condensation of $\alpha$-hydroxyketones $\mathbf{1 a}, \mathbf{b}$ with cyanoacetamides $\mathbf{2 a - d}$ in the presence of a catalytic amount of sodium methoxide afforded 2-imino-2,5-dihydrofuran-3-carboxamides 3a-f that, without isolation, were hydrolized to 2,5-dihydro-2-oxofuran-3-carboxamides $\mathbf{4 a - f}$ in good yields. The products $\mathbf{4 a - f}$ are unsubstituted or substituted at the carboxamide nitrogen atom.

Several syntheses of 2,5-dihydro-2-oxofuran-3-carboxamides have been reported in the literature [23-30]. These authors have previously synthesized compounds 4a-f by related reactions [24-29]. The key step in the current synthesis is the preparation of 2,5-dihydro-2-oxofuran3-carboxamides 4a-f by Knoevenagel condensation of compounds 1a,b with 2a-d. After hydrolysis of the resultant product 3a-f, without isolation, the overall yield of this one-pot two-step synthesis is $74-79 \%$. This method is simpler and more convenient than the methods described earlier.

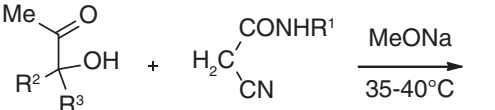

$$
\begin{aligned}
& \text { 2a-d }
\end{aligned}
$$

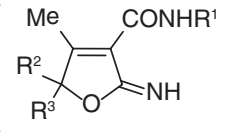

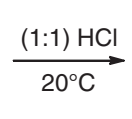

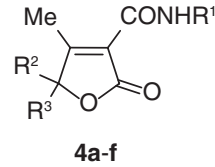

$4 a-f$ 


\section{Experimental}

\section{General procedure for $4 a-f$}

A mixture of an $\alpha$-hydroxyketone $\mathbf{1 a}, \mathbf{b}(10 \mathrm{mmol})$, a cyanoacetamide 2a-d (10 mmol), and sodium methoxide (1 mmol) in absolute methanol $\left(15 \mathrm{~mL}\right.$ ) was heated at $35-40^{\circ} \mathrm{C}$ for $5 \mathrm{~h}$. After concentration, the residue was acidified with (1:1) aqueous $\mathrm{HCl}$ to $\mathrm{pH}$ 1-2 and kept at room temperature for $24 \mathrm{~h}$. The solution was extracted with ethyl ether $(3 \times 20 \mathrm{~mL})$, and the combined organic layers were dried with anhydrous $\mathrm{Na}_{2} \mathrm{SO}_{4}$, filtered, and concentrated. The product $\mathbf{4 a - f}$ was crystallized as indicated below.

4,5,5-Trimethyl-2,5-dihydro-2-oxofuran-3-carboxamide (4a) Yield $77 \%$; mp $125-126^{\circ} \mathrm{C}$ (from petroleum ether), Refs. [23, 24] mp $125-126^{\circ} \mathrm{C}$.

$\mathrm{N}$-Methyl-(4,5,5-trimethyl-2,5-dihydro-2-oxofuran)-3-carboxamide (4b) Yield $79 \%$; $\mathrm{mp} 65-66^{\circ} \mathrm{C}$ (from petroleum ether), Refs. [23, 24] $\mathrm{mp} 65-66^{\circ} \mathrm{C}$.

\section{References}

[1] Ortega, J. J.; Zubia, E.; Ocana, J. M.; Naranjo, S.; Salva, J. New rubrolides from the Ascidian Synoicum blochmanni. Tetrahedron 2000, 56, 3963-3967.

[2] Surivet, J. P.; Vatele, J. M. Concise total synthesis of (+)-goniofufurone and goniobutenolides A and B. Tetrahedron Lett. 1996, 37, 4373-4376.

[3] Jung, J. H.; Pummangura, S.; Chaichantipyuth, S.; Patarapanich, C.; Fanwick, P. E.; Chang, C. J.; McLaughlin, J. L. New bioactive heptenes from Melodorum fruticosum (Annonaceae). Tetrahedron 1990, 46, 5043-5054.

[4] Dawidson, B. S.; Ireland, C. M. Lissoclinolide, the first non-nitrogenous metabolite from a Lissoclinum tunicate. J. Nat. Prod. 1990, 53, 1036-1038.

[5] Miao, S.; Andersen, R. J.; Rubrolides, A.-H. Metabolites of the colonial tunicate Ritterella rubra. J. Org. Chem. 1991, 56, 6275-6280.

[6] Bohlmann, F.; Brindopke, G.; Rastogi, R. Hirsutinolides and other sesquiterpene lactones from Vernonia species. Phytochemistry 1982, 21, 695-699.

[7] Avetisyan, A. A.; Dangyan, M. T. Chemistry of $\alpha, \beta$-butenolides. Usp. Khim. 1977, 46, 1250-1278.

[8] Avetisyan, A. A.; Tokmadzhyan, G. G. Chemistry of $\Delta^{\beta, \gamma-}$ butenolides. Khim. Geterotsikl. Soedin. 1987, 6, 723-739.

[9] Avetisyan, A. A.; Tokmajyan, G. G. Biologically active derivatives of 2-buten- and 3-buten-4-olides. Chem. J. Arm. 1993, 46, 219-236.

[10] Marshallin, P. G. In Chemistry of Carbon Compounds; Rodd, E. H., Ed. Elsevier: New York, 1970; Vol. IID, chapter 17.

[11] Siddall, J. B. US Patent 1972, 3700694. Chem. Abstr. 1973, 78, 43254.

[12] Payne, G. B. US Patent 1965, 3177227. Chem. Abstr. 1965, 63, $6866 \mathrm{e}$.

[13] Laduwahetty, T. Saturated and unsaturated lactones. Contemp. Org. Synth. 1995, 2, 133-149.
N-Benzyl-(4,5,5-trimethyl-2,5-dihydro-2-oxofuran)-3-carboxamide (4c) Yield $76 \% ; \mathrm{mp} 84-85.5^{\circ} \mathrm{C}$ (from petroleum ether), Ref. [23] $\mathrm{mp} 86-88^{\circ} \mathrm{C}$ (from petroleum ether), Ref. [24] $\mathrm{mp} 84-85^{\circ} \mathrm{C}$, Ref. [30] $\mathrm{mp} 83-84^{\circ} \mathrm{C}$.

$\mathrm{N}$-Phenyl-(4,5,5-trimethyl-2,5-dihydro-2-oxofuran)-3-carboxamide (4d) Yield $75 \% ; \mathrm{mp} 97-98^{\circ} \mathrm{C}$ (from petroleum ether), Ref. [23] mp $96.5-98^{\circ} \mathrm{C}$.

4-Methyl-5,5-pentamethylene-2,5-dihydro-2-oxofuran-3-carboxamide (4e) Yield $79 \%$; mp $161-163^{\circ} \mathrm{C}$ (from octane), Ref. [23] mp $161-162.5^{\circ} \mathrm{C}$.

$N$-Methyl-(4-methyl-5,5-pentamethylene-2,5-dihydro-2-oxofuran)-3-carboxamide (4f) Yield $79 \%$; mp $108-109^{\circ} \mathrm{C}$ (from octane), Ref. [23] mp 108-109 ${ }^{\circ} \mathrm{C}$.

Received January 17, 2014; accepted March 9, 2014

[14] Collins, I. Saturated and unsaturated lactones. Contemp. Org. Synth. 1996, 3, 295-321.

[15] Larock, R. C.; Reifling, B. Mercury in organic chemistry. 12. Synthesis of.beta.-chloro-DELTA...alpha.,.,beta.-butenolides via mercuration-carbonylation of propargylic alcohols. J. Org. Chem. 1978, 43, 131-137 (and references therein).

[16] Caine, D.; Stephen, F.; Uckachukawa, V. C. Synthesis of (.+-.)umbelactone. J. Org. Chem. 1983, 48, 740-741.

[17] Desmond, R.; Dolling, U.; Marcune, B.; Tillyer, R.; Tschaen, D. World Patent 1996, W096/08482. Chem. Abstr. 1996, 25, P86474.

[18] Rao, Y. S. Chemistry of butenolides. Chem. Rev. 1964, 64, 353-388.

[19] Rao, Y. S. Recent advances in the chemistry of unsaturated lactones. Chem. Rev. 1976, 76, 625-694.

[20] Garzelli, R.; Samaritani, S.; Malanga, C. 2,5-Dimethoxy2,5-dihydrofuran chemistry: a new approach to $2(5 \mathrm{H})$-furanone derivatives. Tetrahedron 2008, 64, 4183-4186 (and references therein).

[21] De Souza, M. V. N. The furan-2(5H)-ones: recent synthetic methodologies and its application in total synthesis of natural products. Mini-Rev. Org. Chem. 2005, 2, 139-145 (and references therein).

[22] Bellina, F.; Rossi, R. Mucochloric and mucobromic acids: inexpensive, highly functionalized starting materials for the selective synthesis of variously substituted $2(5 \mathrm{H})$-furanone derivatives, sulfur- or nitrogen-containing heterocycles and stereodefined acyclic unsaturated dihalogenated compounds. Curr. Org. Chem. 2004, 8, 1089-1103.

[23] Avetisyan, A. A.; Tatevosyan, G. E.; Dangyan, M. T. Investigations in the field of unsaturated lactones. The reaction of 3-carbethoxy-4-methyl-5,5-dialkyl- $\Delta^{3}$-butenolides with amines. Arm. Chem. J. 1971, 24, 688-693. 
[24] Avetissyan, A. A.; Karapetyan, L. V. The synthesis of new 2-iminoderivatives of 2,5-dihydrofurans and their chemical transformations. Synth. Commun. 2009, 39, 7-19.

[25] Avetisyan, A. A.; Karapetyan, L. V.; Tadevosyan, M. D. Synthesis of 2-imino-4-vinyl-2,5-dihydrofuran-3-carboxamides and some their chemical transformations. Russ. J. Org. Chem. 2009, 45, 1031-1035.

[26] Avetisyan, A. A.; Karapetyan, L. V. Synthesis and chemical transformations of N-cyclohexyl-2-imino-4-methyl-5,5-pentamethylene-2,5-dihydrofuran-3-carboxamide. Russ. J. Org. Chem. 2009, 45, 1578-1580.

[27] Avetisyan, A. A.; Karapetyan, L. V. Synthesis of new heterocyclic derivatives of 2-imino-2,5-dihydrofurans and some of their chemical transformations. Chem. Heterocycl. Compd. 2010, 46, 15-19.

[28] Avetisyan, A. A.; Karapetyan, L. V.; Tadevosyan, M. D. Synthesis and chemical transformations of novel functionalized 2-imino2,5-dihydrofurans. Russ. Chem. Bull. 2010, 59, 974-976.

[29] Avetisyan, A. A.; Karapetyan, L. V. Synthesis and chemical transformations of bis-2-imino-2,5-dihydrofurans. Chem. Heterocycl. Compd. 2013, 48, 1613-1620.

[30] Cheikh, N.; Bar, N.; Choukchou-Braham, N.; Mostefa-Kara, B.; Lohier, J. F.; Sopkova, J.; Villemin, D. Efficient synthesis of new butenolides by subsequent reactions: application for the synthesis of original iminolactones, bis-iminolactones and bis-lactones. Tetrahedron 2011, 67, 1540-1551. 